TITLE:

\title{
Time-domain measurement of picosecond light-pulse propagation in a two-dimensional photonic crystal-slab waveguide
}

\section{$\operatorname{AUTHOR}(S):$}

Asano, T; Kiyota, K; Kumamoto, D; Song, BS; Noda, $\mathrm{S}$

\section{CITATION:}

Asano, $T$...[et al]. Time-domain measurement of picosecond light-pulse propagation in a two-dimensional photonic crystal-slab waveguide. APPLIED PHYSICS LETTERS 2004, 84(23): 4690-4692

\section{ISSUE DATE:}

2004-06-07

URL:

http://hdl.handle.net/2433/50164

\section{RIGHT:}

Copyright 2004 American Institute of Physics. This article may be downloaded for personal use only. Any other use requires prior permission of the author and the American Institute of Physics. 


\title{
Time-domain measurement of picosecond light-pulse propagation in a two-dimensional photonic crystal-slab waveguide
}

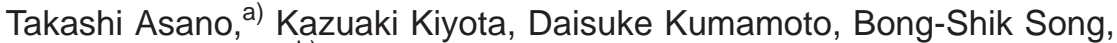 \\ and Susumu Nodab) \\ Department of Electronic Science and Engineering, Kyoto University, Kyoto 615-8510, Japan
}

(Received 6 January 2004; accepted 15 April 2004; published online 19 May 2004)

\begin{abstract}
The optical properties of line-defect waveguides in two-dimensional photonic crystal slabs are investigated using picosecond light pulses. Time-domain waveforms of the light pulse propagating through the waveguide are successfully observed using an autocorrelation method. The group velocity of the waveguide is directly determined from the group delay time for light pulses reflected back and forth along the waveguide. A small group velocity of one-twentieth the speed of light in vacuum is observed at a frequency near the edge of the waveguide mode. The frequency dependence of the group velocity is also measured, and the group-velocity dispersion is found to be larger than that of normal single-mode optical fibers by a factor of $10^{4}-10^{5}$. (C) 2004 American Institute of Physics. [DOI: 10.1063/1.1760224]
\end{abstract}

A photonic crystal (PC) is an optical material with a periodically varying refractive index, in which a gap in frequency, known as a photonic band gap (PBG), is created in the photonic mode spectrum. It is expected that the control of photons in PCs can be realized by introducing artificial defects that locally disturb the PBG. Line and point defects in PCs have previously been utilized as waveguides ${ }^{1-3}$ and cavities, ${ }^{4-8}$ respectively. Unique characteristics have been predicted, such as a low group velocity in line-defect waveguides and the ability to trap photons for long periods of time in point-defect cavities. Although such characteristics result from the dynamics of light pulses or wavepackets within the defects, experimental results reported so far in this field have been based on measurements using continuouswave light. ${ }^{8,9}$ Such wavelength-domain measurements are considered to be important, but do not give a fully adequate description of the dynamic behavior of photons in artificial PC defects. Time-domain experiments utilizing considerably shorter light pulses are indispensable for this purpose. For bulk PCs containing no artificial defects, there have been a few reports of the direct measurement of the propagation of light pulses. ${ }^{10-14}$ The change in shape or the time delay of light pulses propagated through three-dimensional ${ }^{10,11}$ and two-dimensional $^{12,13}$ PCs have been investigated. However, there are no reports on the dynamics of wavepackets in artificial PC defects, the study of which is of great importance for the development of practical devices. In this report, we present the results of time-domain measurements of the propagation of picosecond light pulses along line-defect waveguides in a two-dimensional PC slab. The group delay for pulse propagation along the waveguide is successfully obtained, from which the group velocity is directly determined. The group-velocity dispersion is also discussed.

We investigated line-defect waveguides that were introduced in air-bridge-type two-dimensional PC slabs in which air holes form triangular lattice patterns, shown schemati-

\footnotetext{
${ }^{a)}$ Electronic mail: tasano@kuee.kyoto-u.ac.jp

${ }^{b)}$ Electronic mail: snoda@kuee.kyoto-u.ac.jp
}

cally in Fig. 1(b). Three samples with different lattice constants $a(420,415$, and $410 \mathrm{~nm})$ were prepared. Silicon-oninsulator (SOI) substrates were used for the fabrication of samples, which consisted of a $0.25-\mu \mathrm{m}$-thick Si slab on top of a 1.5 - $\mu$ m-thick $\mathrm{SiO}_{2}$ layer formed on a $700-\mu$ m-thick $\mathrm{Si}$ substrate. Electron-beam lithography and induction-coupled plasma-reactive ion-etching techniques were used to construct the PC patterns on the upper Si slabs. The radius of the air holes was $120 \mathrm{~nm}$ and the line-defect waveguide was formed by removing a row of air holes along the $\Gamma-J$ direction. After the PC patterns had been created, an air-bridgetype structure was formed by removing the underlying $\mathrm{SiO}_{2}$ layers using a selective wet-etching technique. The samples were then cleaved, giving waveguides with lengths $(l)$ of $\sim 250 \mu \mathrm{m}$. Optical measurements were carried out using a passive mode-lock fiber laser as a light source, in which the time width and center wavelength $\left(\lambda_{c}\right)$ of the pulses were tunable in the range $0.6-3.0 \mathrm{ps}$ and $1530-1555 \mathrm{~nm}$, respectively. The repetition frequency of the laser was $50 \mathrm{MHz}$ and hence the interval between the output pulses was $20 \mathrm{~ns}$. Light pulses were injected into the waveguide through an objective lens, and pulses emitted from the opposite end of the waveguide were collected by a second objective lens. The collected light was collimated into a single-mode fiber and amplified by an Er-doped fiber amplifier. An optical autocorrelation method using second harmonic generation was utilized to analyze the time-domain waveform of the output light.

Typical results of our measurements are shown in Fig. 1 (a); in this case, the lattice constant of the sample is 420 $\mathrm{nm}$, and the time width and $\lambda_{c}$ of the input light pulses are $0.6 \mathrm{ps}$ and $1530 \mathrm{~nm}$, respectively. The obtained waveform is symmetric about the time origin as it is detected by the autocorrelation method. The figure shows a pulse train with decreasing intensities and constant intervals of $\sim 8.5 \mathrm{ps}$ between pulses, which is much smaller than the pulse interval of the light source $(20 \mathrm{~ns})$. The observed pulse train can be explained as follows [see Figs. 1(b) and 1(c)]. The first pulse passes directly through the waveguide, and subsequent 


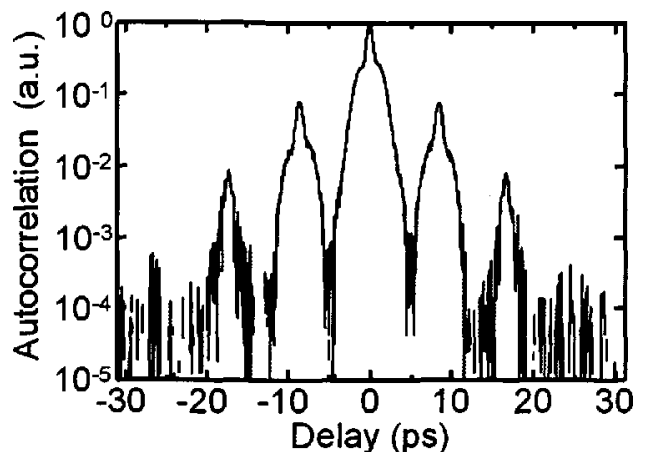

(a)

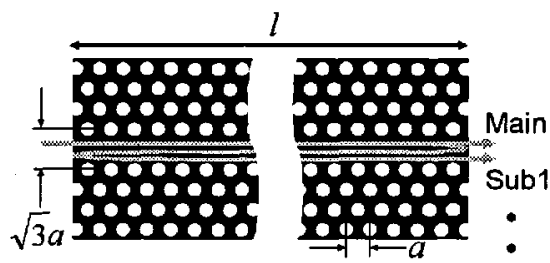

(b)

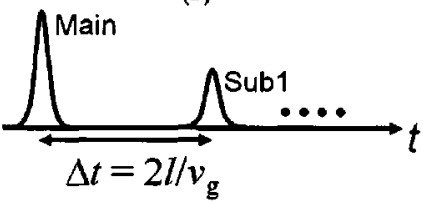

(c)

FIG. 1. (a) Autocorrelation signal of a pulse propagating through a sample with lattice constant $420 \mathrm{~nm}$. The center wavelength and time width of the injected light pulse are $1530 \mathrm{~nm}$ and $0.6 \mathrm{ps}$, respectively; (b) schematic drawing of the sample configuration and propagation path of the light pulse; (c) schematic drawing of the relationship between group velocity and the interval of output pulses.

pulses correspond to those that experience additional roundtrips back and forth along the waveguide due to reflection at the waveguide edges. The interval between individual pulses $(\Delta t)$ represents the group delay for a single roundtrip, and hence the group velocity $\left(v_{g}\right)$ of the waveguide can be directly obtained:

$$
v_{g}=\frac{2 l}{\Delta t}
$$

In the case of Fig. 1, in which the observed pulse interval is $8.5 \mathrm{ps}$ and the waveguide length is $250 \mu \mathrm{m}$, the group velocity is found to be $0.20 c$ ( $c$ is the speed of light in vacuum).

We next investigated the group velocities for samples with different lattice constants $(410-420 \mathrm{~nm})$ by varying the $\lambda_{c}$ of the light pulse between 1530 and $1555 \mathrm{~nm}$. The group velocity is plotted in Fig. 2(a) as a function of the center frequency of the light pulse $(f)$, which has been normalized by the unit frequency of the PC $(c / a)$. Here, we have expanded the normalized frequency range of the measurement by employing the fact that the normalized frequency varies with the lattice constant of the PC, even though the frequency of the light is constant. ${ }^{14}$ It is clearly seen in the figure that the group velocity rapidly decreases as the frequency is reduced. For comparison, the dispersion curve of the waveguide is theoretically calculated using the threedimensional finite-difference time domain (FDTD) method. Figure 2(b) shows the calculated dispersion curve and the dotted line in Fig. 2(a) represents the calculated group velocDownloaded 04 Mar 2008 to 130.54 .110 .22 . Redistribution subject

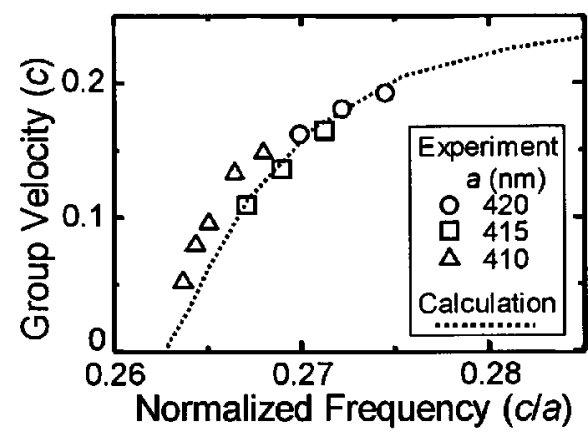

(a)

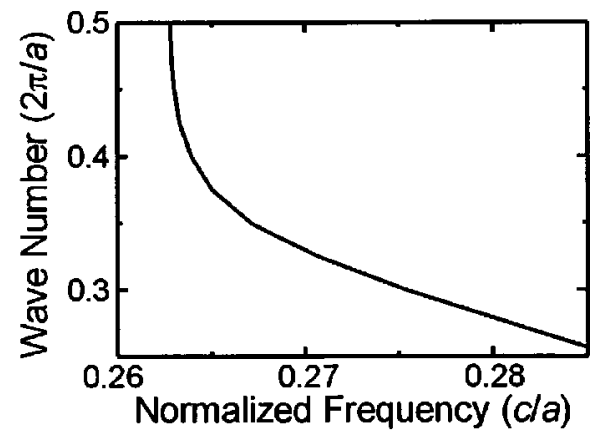

(b)

FIG. 2. (a) Measured group velocities as a function of the normalized frequency of the light pulse (open symbols). The theoretically calculated group velocity is also shown for comparison (dotted line); (b) theoretical dispersion curve of the sample waveguide.

ity, which was obtained from the slope of the dispersion curve. Figure 2(a) shows that the experimental and theoretical group velocities agree well with each other. By comparing Figs. 2(a) and 2(b), it is found that the group velocity becomes small as the frequency approaches the lower edge of the waveguide mode. The time-domain waveform for the propagation of the light pulse with the lowest normalized frequency is shown in Fig. 3. It is seen that the light pulse takes up to 32 ps to complete a roundtrip along a waveguide of length $250 \mu \mathrm{m}$. The corresponding group velocity is the smallest observed in our series of experiments, reaching a minimum value of $0.05 c$.

We have also investigated the group-velocity dispersion (GVD) and the energy loss involved in the propagation. In the case of Fig. 1, in which the frequency of the injected

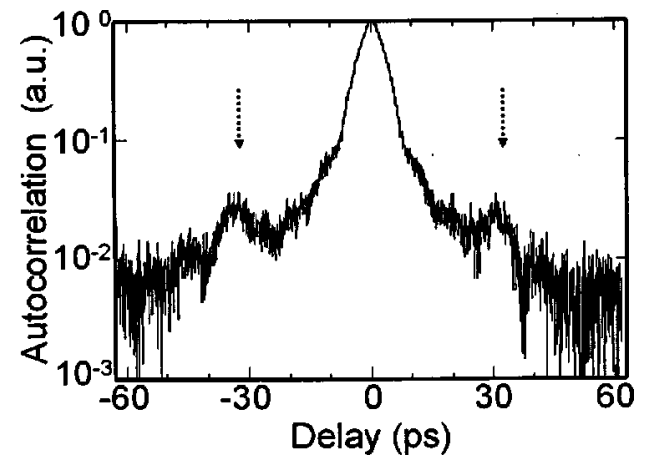

FIG. 3. Autocorrelation signal of a pulse propagating through a sample with lattice constant $410 \mathrm{~nm}$ at a frequency near the lower edge of the waveguide mode. The center wavelength and time width of the injected light pulse are $1555 \mathrm{~nm}$ and $3 \mathrm{ps}$, respectively. The group delay for one roundtrip is $32 \mathrm{ps}$ and the calculated group velocity is as small as $0.05 \mathrm{c}$. 
light pulse is relatively high $(0.275 c / a)$, the pulse shape is maintained for two roundtrips along a $250 \mu \mathrm{m}$ waveguide. This implies that the effect of GVD at $f=0.275 \mathrm{c} / a$ is so small that it cannot be observed under the conditions utilized here (pulse width $=0.6 \mathrm{ps}$ and propagation length $=1 \mathrm{~mm}$ ). As the pulse shape is maintained, the decrease in the pulse intensity, which is $\sim 10 \mathrm{~dB}$ per roundtrip, originates from propagation loss and reflection loss at both ends of the waveguide. The reflection loss calculated by the three-dimensional FDTD method is $\sim 4 \mathrm{~dB}$ per reflection and, as a result, the propagation loss might be roughly estimated as $\sim 2 \mathrm{~dB}$ per $500 \mu \mathrm{m}$. By contrast, in the case of Fig. 3, in which the frequency of the injected light pulse $(f=0.263 \mathrm{c} / \mathrm{a})$ is near the edge of the waveguide mode, the second pulse is broader than the first pulse. Therefore, the deformation of the pulse shape makes it impossible to estimate the intensity loss for the roundtrip. This result implies that GVD has an observable effect on the pulse propagation (pulse width $=\sim 3 \mathrm{ps}$ and propagation length $=500 \mu \mathrm{m}$ ) when the frequency is near the mode edge. The values of the GVD were also evaluated using the derivative of the experimental dispersion curve shown in Fig. 2(a) and ranged from $1 \times 10^{5} \mathrm{ps} / \mathrm{nm} / \mathrm{km}$ (at $f$ $=0.275 \mathrm{c} / \mathrm{a})$ to $4 \times 10^{6} \mathrm{ps} / \mathrm{nm} / \mathrm{km}($ at $f=0.263 \mathrm{c} / a)$. The observed GVDs are four to five orders of magnitude larger than those in conventional single-mode optical fibers (typically 17 $\mathrm{ps} / \mathrm{nm} / \mathrm{km}$ at $1550 \mathrm{~nm})$.

In summary, we have studied the propagation of picosecond light pulses along line-defect waveguides in twodimensional PC slabs. The group velocity of the waveguide has been directly determined from the group-delay time for reflected light pulses that undergo roundtrips back and forth along the waveguide. A small group velocity of $0.05 c$ has been observed. The frequency dependence of the group velocity has also been measured and the group velocity disper- sion was found to be larger than that of conventional singlemode fibers by a factor of $10^{4}-10^{5}$. Our results indicate that time-domain measurements are useful for the investigation of the dynamic characteristics of artificial defects in PCs. We expect that line-defect waveguides in two-dimensional PC slabs will find applications in the near future as dynamic pulse-control devices such as ultracompact delay lines and dispersion compensators.

This work was partly supported by Core Research for Evolution Science and Technology (CREST), Japan Science and Technology Corporation (JST) and Grant-in-Aids for Scientific Research from the Ministry of Education, Culture, Sport, Science, and Technology of Japan.

${ }^{1}$ A. Chutinan and S. Noda, Phys. Rev. B 62, 4488 (2000).

${ }^{2}$ A. Chutinan and S. Noda, Jpn. J. Appl. Phys., Part 1 39, 2353 (2000).

${ }^{3}$ S. G. Jonson, P. R. Villeneuve, S. Fan, and J. D. Joannopoulos, Phys. Rev. B 62, 8212 (2000).

${ }^{4}$ S. Noda, A. Chutinan, and M. Imada, Nature (London) 407, 608 (2000).

${ }^{5}$ O. Painter, J. Vuckovic, and A. Scherer, J. Opt. Soc. Am. B 16, 275 (1999).

${ }^{6}$ T. Asano, M. Mochizuki, S. Noda, M. Okano, and M. Imada, J. Lightwave Technol. 21, 1370 (2003).

${ }^{7}$ K. Srinivasan et al., Appl. Phys. Lett. 83, 1915 (2003).

${ }^{8}$ Y. Akahane, T. Asano, B. S. Song, and S. Noda, Nature (London) 425, 944 (2003).

${ }^{9}$ M. Notomi, K. Yamada, A. Shinya, J. Takahashi, C. Takahashi, and I. Yokohama, Phys. Rev. Lett. 87, 253902 (2001).

${ }^{10}$ A. Imhof, W. L. Vos, R. Sprik, and A. Lagendijk, Phys. Rev. Lett. 83, 2942 (1999).

${ }^{11}$ T. Tanaka, S. Noda, A. Chutinan, T. Asano, and N. Yamamoto, J. Opt. Quantum Electron. 34, 37 (2002).

${ }^{12}$ K. Inoue, N. Kawai, Y. Sugimoto, N. Charlsson, N. Ikeda, and K. Asakawa, Phys. Rev. B 65, 121308(R) (2002).

${ }^{13}$ M. C. Netti, C. E. Finlayson, J. J. Baumberg, M. D. B. Charlton, M. E. Zoorob, J. S. Wilkinson, and G. Parker, Appl. Phys. Lett. 81, 3927 (2002).

${ }^{14}$ B. S. Song, S. Noda, and T. Asano, Science 300, 1537 (2003). 